\title{
Compi: a framework for portable and reproducible pipelines
}

\author{
Hugo López-Fernández ${ }^{1,2,3}$, Osvaldo Graña-Castro ${ }^{4}$, Alba Nogueira-Rodríguez ${ }^{1,2,3}$, Miguel Reboiro-Jato ${ }^{1,2,3}$, Daniel \\ Glez-Peña ${ }^{\text {Corresp. 1, 2, } 3}$ \\ ${ }^{1}$ ESEI: Escuela Superior de Ingeniería Informática, University of Vigo, Ourense, Galicia, Spain \\ 2 CINBIO - Centro de Investigaciones Biomédicas, University of Vigo, Vigo, Galicia, Spain \\ 3 SING Research Group, Galicia Sur Health Research Institute (IIS Galicia Sur). SERGAS-UVIGO, Vigo, Galicia, Spain \\ 4 Bioinformatics Unit, Structural Biology Programme, Spanish National Cancer Research Centre (CNIO), Madrid, Madrid, Spain \\ Corresponding Author: Daniel Glez-Peña \\ Email address: dgpena@uvigo.es
}

Compi is an application framework to develop end-user, pipeline-based applications with a primary emphasis on: (i) user interface generation, by automatically generating a command-line interface based on the pipeline specific parameter definitions; (ii) application packaging, with compi-dk, which is a version-control-friendly tool to package the pipeline application and its dependencies into a Docker image; and (iii) application distribution provided through a public repository of Compi pipelines, named Compi Hub, which allows users to discover, browse and reuse them easily. By addressing these three aspects, Compi goes beyond traditional workflow engines, having been specially designed for researchers who want to take advantage of common workflow engine features (such as automatic job scheduling or logging, among others) while keeping the simplicity and readability of shell scripts without the need to learn a new programming language. Here we discuss the design of various pipelines developed with Compi to describe its main functionalities, as well as to highlight the similarities and differences with similar tools that are available. An open-source distribution under the Apache 2.0 License is available from GitHub (https://github.com/sing-group/compi). Documentation and installers are available from https://www.sing-group.org/compi. A specific repository for Compi pipelines is available from Compi Hub (https://www.sing-group.org/compihub). 


\section{Compi: a framework for portable and reproducible 2 pipelines}

3

4 Hugo López-Fernández ${ }^{1,2,3}$, Osvaldo Graña-Castro ${ }^{4}$, Alba Nogueira-Rodríguez ${ }^{1,2,3}$, Miguel

5 Reboiro-Jato ${ }^{1,2,3}$, Daniel Glez-Peña ${ }^{1,2,3}$

6

$7 \quad{ }^{1}$ ESEI: Escuela Superior de Ingeniería Informática, University of Vigo, Ourense, Galicia, Spain

$8 \quad{ }^{2}$ CINBIO - Centro de Investigaciones Biomédicas, University of Vigo, Vigo, Galicia, Spain

$9 \quad{ }^{3}$ SING Research Group, Galicia Sur Health Research Institute (IIS Galicia Sur). SERGAS-

10 UVIGO, Vigo, Galicia, Spain

$11{ }^{4}$ Bioinformatics Unit, Structural Biology Programme, Spanish National Cancer Research Centre

12 (CNIO), Madrid, Madrid, Spain

14 Corresponding Author:

15 Daniel Glez-Peña ${ }^{1,2,3}$

16 ESEI: Escuela Superior de Ingeniería Informática, University of Vigo, Ourense, Galicia, Spain

17 Email address: dgpena@uvigo.es

18

19 


\section{Abstract}

21 Compi is an application framework to develop end-user, pipeline-based applications with a

22

23

24

25

26

27

28

29

30

31

32

33

34

35

36

37

38

39

40

41

42

43

44

45

46

47

48

49

50

51

52

53

54

55

56

57

58

59

primary emphasis on: $(i)$ user interface generation, by automatically generating a command-line interface based on the pipeline specific parameter definitions; (ii) application packaging, with compi-dk, which is a version-control-friendly tool to package the pipeline application and its dependencies into a Docker image; and (iii) application distribution provided through a public repository of Compi pipelines, named Compi Hub, which allows users to discover, browse and reuse them easily. By addressing these three aspects, Compi goes beyond traditional workflow engines, having been specially designed for researchers who want to take advantage of common workflow engine features (such as automatic job scheduling or logging, among others) while keeping the simplicity and readability of shell scripts without the need to learn a new programming language. Here we discuss the design of various pipelines developed with Compi to describe its main functionalities, as well as to highlight the similarities and differences with similar tools that are available. An open-source distribution under the Apache 2.0 License is available from GitHub (https://github.com/sing-group/compi). Documentation and installers are available from https://www.sing-group.org/compi. A specific repository for Compi pipelines is available from Compi Hub (https://www.sing-group.org/compihub).

\section{Introduction}

Bioinformatics units routinely deal with massive data analyses, which require combining multiple sequential or parallel steps using specific software tools (Perkel, 2019). Many of these computational pipelines are published regularly in the form of protocols, best practices or even fully runnable pipelines. They implement all the required steps and dependencies in order to ensure the reproducibility of the analyses and facilitate job automation (Grüning et al., 2018). Thus, scientific computational pipelines must provide three key features: reproducibility, portability and scalability. Container technologies such as Docker or Singularity are the most widely used tools to ensure that pipelines run in stable environments (i.e. always using the exact same version of pipeline dependencies) and make it easy to run the pipeline on multiple hardware platforms (e.g. workstations or cloud infrastructures), enforcing reproducibility and portability. Moreover, scalable pipelines must support running on HPC (High Performance Computing) resources using cluster management and job scheduling systems such as Slurm or SGE.

For the above reasons, a wide variety of workflow management systems have been released in recent years that address these issues in different ways. Tools with graphical user interfaces, such as Galaxy (Afgan et al., 2018), are designed for scientists with little or no programming experience, although such tools can be difficult to set up and configure. Furthermore, commandline based applications such as Nextflow (Di Tommaso et al., 2017), Snakemake (Köster \& Rahmann, 2012), or SciPipe (Lampa et al., 2019), provide feature-rich workflow engines oriented to bioinformaticians with medium-to-high programming skills. The Common Workflow Language (CWL; https://www.commonwl.org/) definition represents another alternative, since it

Peer] Comput. Sci. reviewing PDF | (CS-2021:01:57469:1:1:NEW 3 May 2021) 
60

61

62

63

64

65

66

67

68

69

70

71

72

73

74

75

76

77

78

79

80

81

82

83

84

85

86

87

88

89

90

91

92

93

\section{4}

95

96

97

98

99

defines a specification and offers a reference implementation, but does not provide a complete framework (Leipzig, 2017). Other frameworks like Galaxy or Taverna made significant progress to support the execution of workflows defined in CWL and other tools allow to export their workflows into CWL (e.g. Snakemake) or import them from CWL.

Despite the existence of such remarkable workflow management systems, scientists with basic scripting skills (e.g. able to create shell scripts invoking command-line tools) but lacking advanced programming skills (e.g. knowledge of programming languages such as Python or Go), are usually overwhelmed due to the high complexity of these systems, and could be hampered to use or create their own workflows. In this sense, tools such as Bpipe (Sadedin, Pope \& Oshlack, 2012) help to assemble shell scripts into workflows to aid in job automation, logging and reproducibility.

Compi is specially designed for researchers who want to take advantage of common workflow engine features (e.g. automatic job scheduling, restart from point of failures, etc.) while maintaining the simplicity and readability of shell scripts, without the need to learn a new programming language. Nevertheless, Compi also incorporates several features that meet the needs of the most advanced users, such as support for multiple programming languages or advanced management and control of workflow execution. In this sense, Compi is more than a workflow engine, it was created as an application framework for developing pipeline-based enduser applications by providing: (i) automatic user interface generation - generates a classical command-line interface (CLI) for the entire pipeline based on its parameter specifications; (ii) application packaging - provides a version-control-friendly mechanism to package the pipeline application and its dependencies into a Docker image; and (iii) application distribution, supported by Compi Hub (Nogueira-Rodríguez et al., 2021), a public repository where researchers can easily and freely publish their Compi pipelines and related documentation, making them available for other researchers.

Compi has been adopted by our research group to create pipelines for multiple research projects where other systems were not appropriate enough (e.g. the creation of complex pipelines for phylogenomics or training of models based on deep learning for image classification). Likewise, pipelines developed with Compi in collaboration with other research groups have already been published. These are the cases of Metatax, a pipeline to analyze biological samples based on $16 \mathrm{~S}$ rRNA gene sequencing (Graña-Castro et al., 2020a,b), FastScreen, a pipeline for inferring positive selection in large viral datasets (López-Fernández et al., 2020), and GenomeFastScreen, an extension of the FastScreen pipeline (López-Fernández et al., 2021).

\section{Materials \& Methods}

This section depicts the most relevant technical details regarding the implementation of Compi. An open-source distribution under the Apache 2.0 License is available from GitHub (https://github.com/sing-group/compi). Documentation and installers are available from https://www.sing-group.org/compi. 
100

101

102

103

104

105

106

107

108

109

110

111

112

113

114

115

116

117

118

119

120

121

122

123

124

125

126

127

128

129

130

131

132

133

134

135

136

137

138

\section{$X M L$ for pipeline definition}

Compi pipelines are defined in a single XML (eXtensible Markup Language) file that includes (i) tasks and dependencies between them, (ii) pipeline input parameters that are forwarded to tasks, and (iii) metadata, including parameters and tasks descriptions, which are useful for automatic generation of the user interface, as well as for pipeline documentation. We have chosen XML instead of JSON, YAML, or a custom DSL (Domain Specific Language) to reconcile the various requirements simultaneously. First, we wanted a high degree of interoperability. XML, JSON, and YAML can be easily generated and parsed in almost any programming language, although YAML can pose portability issues in some cases as YAML parsers in different languages can produce different results. In contrast, a DSL (Di Tommaso et al., 2017) is less interoperable, being difficult to produce or consume from languages other than the one on which the DSL is based. Second, XML is appropriate for dealing with long chunks of text, such as the embedded source code for pipeline tasks. Since Compi is language agnostic, these tasks can be defined in any programming language. Thanks to the XML CDATA (character data) blocks, which allow declaring a section of the XML that should not be parsed as XML, it is possible to include source code without any alteration. Embedding task code in JSON is virtually not feasible, as tabs and line breaks, which are key characters in languages like Python, must be escaped in JSON files. In YAML, however, it is easier thanks to its multiline literals. In DSLs it depends on whether the programming language the DSL is based on allows you to declare multiline strings easily, as Python does. Third, XML is easy to validate syntactically and semantically through schemas, which are also present in JSON and YAML. DSLs take advantage of the language parser the DSL is based on. Fourth, for security reasons, since Compi is designed to run pipelines defined by third-party programmers, YAML is a less secure language in this regard, as runnable code could be embedded in fields that were not intended for this purpose (see https://www.arp242.net/yaml-config.html). Fifth comes readability. YAML is the clearer winner in this regard, as readability is the key feature of this format. Although XML is less readable than YAML, the comparison with JSON and DSLs is a more subjective matter. Based on these five requirements, the choice was between XML and YAML: while XML is more secure and portable, YAML is more readable. Finally, we selected XML, prioritizing its security, portability and popularity. Also, while writing XML files could be verbose, YAML is syntactically aware of whitespace, which is a welcome feature in the Python community, but still debated outside of it.

\section{The workflow execution engine}

The workflow execution engine of Compi is implemented purely in Java and is responsible for multi-thread task scheduling, monitoring, and standard error and output logging of tasks. First, it computes the DAG (Direct Acyclic Graph) of the task dependencies, since a task may depend on a set of tasks that must be run before the given task. Right after starting, or whenever a task

finishes its execution, the engine reacts and all the tasks that can be run, i.e. the tasks whose 
139 dependencies are now complete, are sent to a worker thread pool that has a parameterizable size.

140 When there are no more tasks to run, the pipeline execution ends.

141 Every time a task is about to run and there is a free thread in the pool, a new subprocess is

142 spawned by invoking the system Bash interpreter to execute the task script. Pipeline parameters

143 are passed to the task script through environment variables, which is a robust, standard

144 mechanism with two main benefits. On the one hand, environment variables are easily accessed

145 via "\$variable_name" or more complex expressions, so pipeline parameters are available directly

146 within the task script. On the other hand, this allows Compi to pass parameters to scripts written

147 in languages other than Bash, because virtually all programming languages give access to

148 environment variables.

149 The way to execute languages differently from Bash is through task interpreters. Any task can

150 have a task interpreter defined in the pipeline specification, which is an intermediate, user-

151 defined bash script intended to take the task script as input and call an interpreter from a different

152 programming language.

153 Moreover, it is possible to define task runners, which are the same concept as interpreters, but

154 with a different purpose. They are not defined within the pipeline specification, but rather at

155 execution time, and are intended to tailor task execution to specific computing resources, without

156 modifying the pipeline itself. In this way, the workflow definition is decoupled from the

157 workflow execution, making it possible to change the way tasks are executed without modifying

158 the workflow XML file. For instance, if the tasks must be run in a cluster environment such as

159 SGE or Slurm, computations must be initiated via a submission command (qsub in SGE or srun

160 in Slurm). Task runners intercept task execution by changing the default Bash interpreter with

161 queue submissions.

162

\section{Compi project architecture}

164 Compi comprises three main modules. The most important is the core module, which contains

165

166

167

168

169

170

171

172

173

174

175

176

177

178

the workflow execution engine, with its main data structures. On top of it, there are two additional modules. On the one hand, the cli (command-line interface) module contains the command-line user interface for running pipelines, which generates a specific pipeline application tailored for pipeline tasks and parameters. On the other hand, the $d k$ (Development Kit) module allows to create a portable application in the form of a Docker image and publishing the pipelines at Compi Hub.

\section{Compi Hub}

As discussed above, Compi Hub is a public repository where Compi pipelines can be published. The Compi Hub front-end was implemented using the Angular v7 web application framework, while the back-end was implemented using TypeScript and offers a RESTful API that supports all the functionality of the front-end. This REST API is also used by the compi-dk tool to allow pipeline developers to publish their pipelines from the command line. The Compi Hub back-end runs in a Node.js server and uses a MongoDB database to store the data.

PeerJ Comput. Sci. reviewing PDF | (CS-2021:01:57469:1:1:NEW 3 May 2021) 


\section{Results}

181 Compi can be seen as an ecosystem comprising: (i) compi, the workflow engine with a

182

183

184

185

186

187

188

189

190

191

192

193

194

195

196

197

198

199

200

201

202

203

204

205

206

207

208

209

210

211

212

213

214

215

216

217

218

command-line user interface to control the pipeline execution, (ii) compi-dk, a command-line tool to assist in the development and packaging of Compi-based applications, and (iii) Compi Hub, a public repository of Compi pipelines that allows users to easily discover, navigate and reuse them (Nogueira-Rodríguez et al., 2021). Figure 1 illustrates the development and deployment lifecycle of Compi-based applications. A Compi-based application is a CLI application in which a user can run a pipeline in whole or in part by providing only the parameters of each task. This CLI displays all parameter names and descriptions following standard CLI conventions to help users use the pipeline application more easily. Compi helps pipeline developers by automatically determining the parameters required by a pipeline. This implies, on the one hand, that comprehensive help is generated for the user on how to use the application and, on the other hand, that the application parameters are automatically parsed when the pipeline is executed. This is complemented by several built-in features added to the CLI application, such as an advanced task execution control, allowing to run only specific tasks in a pipeline, or log management support per task. Therefore, building a CLI application from a Compi pipeline is a straightforward process that allows Compi users to focus on pipeline development. The "Pipeline design" section explains how Compi users can design their pipelines.

Once the pipeline is developed, it can take two complementary paths: it can be executed directly with compi (top right of Figure 1) or it can be packaged as a portable end-user CLI application in a Docker image using compi-dk (application packaging area in Figure 1), which can be then executed via Docker. Pipeline execution and dependency management are explained in the "Pipeline execution and dependency management" section, while the application packaging in Docker images is explained in the "Reproducible Application Packaging with Docker" section. When an end-user pipeline application is ready to be published, Compi provides pipeline developers with a distribution platform called Compi Hub, where they can share different versions of their pipelines along with usage documentation, example datasets, parameter values, as well as links of interest (e.g. Docker Hub, Github). Community users can then browse the Compi Hub for pipelines and can explore the helpful documentation generated by the authors of each pipeline, as well as other information automatically generated by the platform, such as an interactive DAG representation of the pipeline. This topic is covered in the "Pipeline distribution via Compi Hub" section.

Finally, it is important to note that compi-dk projects are particularly designed to be compatible with version control systems, as the required configuration and pipeline files are text files. Dependency management via a Dockerfile is key to achieve this, complemented by Compispecific dependency management performed by compi-dk (e.g. the compi executable added to the Docker image of the pipeline). Therefore, a compi-dk project only requires that compi, compi-dk and Docker are installed in order to be built or run. With this feature, Compi gives pipeline

Peer] Comput. Sci. reviewing PDF | (CS-2021:01:57469:1:1:NEW 3 May 2021) 
219 developers the ability to use version control systems to keep their pipeline safe and to version 220 their code.

221

222

223

224

225

226

227

228

229

230

231

232

233

234

235

236

237

238

239

240

241

242

243

244

245

246

247

248

249

250

251

252

253

254

255

256

257

258

\section{Pipeline design}

As explained previously, Compi pipelines are defined in an XML document that includes user parameters, tasks, and metadata. Figure 2 shows an example of a minimal pipeline. This sample pipeline defines two parameters ("name" and "output"), described in the corresponding "params" section, and two tasks ("greetings" and "bye") described in the "tasks" section. The parameter descriptions are used to automatically generate both the user interface and the documentation for the pipeline. In the same way and for the same purpose, tasks are described in the "metadata" section, which allows the pipeline developer to describe them in a human-readable manner. Tasks are defined as "task" elements within the "tasks" section. The main components of a task are: source code, parameters and dependencies. The source code is placed inside a "task" element, and the parameters used by a task are defined within the "params" attribute, and those tasks that the current task depends on are defined in the "after" attribute. For instance, in Figure 2 , the "bye" task, uses the "name" and "output" parameters, and depends on the "greetings" task. Parameter values are passed to the tasks as environment variables, which is a standard method that any programming language gives access to. In this way, the Compi workflow execution engine does not need to process the task code in any way (e.g. to perform variable substitution), guaranteeing the possibility of using any programming language to define the code of the task. In this sense, the task code is written in Bash by default, although other scripting languages (e.g. Python, R, AWK, etc.) can be used under a suitable interpreter through an "interpreter" parameter. For example, in Figure 2, the "bye" task is written in Perl and the "interpreter" parameter indicates how to invoke the Perl interpreter to run the task code.

A special type of tasks are parallel iterative tasks (or loop tasks), defined via "foreach" elements, which spawn multiple parallel processes with the same code on a collection of items. This collection of items is provided by a user-specified source of items, which could be a commaseparated list of values, a range of numbers, the files in a specific directory, a parameter whose value is a list of values separated by commas, or even a custom command whose output lines are taken as items. Each item is available to the task code as an environment variable.

When all spawned tasks have finished, the foreach task ends as well and subsequent dependent tasks can be run. Nevertheless, there are several scenarios in which a pipeline developer can define multiple consecutive foreach tasks intended to iterate over the same collection of independent items. In these scenarios, when one iteration of a loop has finished, the corresponding iteration of the next loop could start without waiting for the entire previous loop to finish. Compi supports this type of interaction between foreach tasks by simply adding the '*', prefix to the task name when declaring the dependency on the "after" attribute. For example, in a scenario where a pipeline must process a set of samples (e.g: "case-1", "case-2", "control-1", "control-2") by performing two consecutive operations: preprocess and analyze, the dependency of the "preprocess" task on the "analyze" task can be prefixed with '*' (i.e after=" "preprocess") 
259

260

261

262

263

264

265

266

267

268

269

270

271

272

273

274

275

276

277

278

279

280

281

282

283

284

285

286

287

288

289

290

291

292

293

294

295

296

297

298

to tell Compi that iterations over the "analyze" loop could start when the corresponding iterations over the "preprocess" loop have finished.

Figure 3 shows an example of this, where two foreach tasks "preprocess" and "analyze" iterate over the same set of items ("samples"). The "analyze" task depends on the "preprocess" task, but at an iteration-level (after =" "preprocess"). Without the "*' prefix, the "analyze" task will only start when the whole "preprocess" task has finished.

\section{Pipeline execution and dependency management}

One of the main features of Compi is the creation of a classic CLI for the entire pipeline based on its parameter specifications to aid users run the pipeline. This CLI is displayed when a user executes "compi run -p pipeline.xml--help", which describes the Compi execution parameters and specification of each pipeline task. Supplementary File 1 shows this CLI for the RNA-Seq Compi pipeline (https://www.sing-group.org/compihub/explore/5d09fb2a1713f3002fde86e2). As Figure 4 illustrates, the execution of Compi pipelines can be controlled using multiple parameters that fall in three main categories: pipeline inputs (i.e. the pipeline definition and its input parameters), logging, and execution control. In Figure 4A, the "compi run" command receives the pipeline definition file explicitly, while the "--params" option indicates that the input parameters must be read from the "compi.params" file. On the other hand, in Figure 4B the pipeline definition file is omitted and compi assumes that the pipeline definition must be read from a file named "pipeline.xml" located in the current working directory. Also, pipeline parameters are passed on the command line after all the "compi run" parameters and separated by the '--' delimiter.

Regarding logging options, both include the "-- logs" option to specify a directory to save the standard (stdout) and error (stderr) outputs of each task along with the specific parameter values used in each execution. They are saved in three different files named with the name of the corresponding task as prefix (e.g. "task-name.out.log ", "task-name.err.log", and "taskname.params"). To avoid unnecessary file creation, Compi does not save task outputs unless the "--logs" option is used. However, it is possible to specify which tasks should be logged using the "--log-only-task" or "--no-log-task" parameters. In addition to the task specific logs, Compi displays its own log messages during pipeline execution. These messages can be disabled by including "--quiet" as shown in Figure 4B. In contrast, in the example given in Figure 4A, the "-show-std-outs" option forces Compi to forward each task log to the corresponding Compi output, which is very useful for debugging purposes during pipeline development.

The third group of options allows to control the execution of the pipeline. For instance, the "-num-tasks" parameter used in Figure 4A sets the maximum number of tasks that can be run in parallel. It is important to note that this is not necessarily equivalent to the number of threads the pipeline will use, as some tasks may use parallel processes themselves. The "--abort-ifwarnings" option, also used in the example in Figure 4A, tells Compi to abort the pipeline execution if there are warnings in the pipeline validation. This is a useful and recommended option for pipeline testing during development, to avoid undesired effects that may arise from 
299

300

301

302

303

304

305

306

307

308

309

310

311

312

313

314

315

316

317

318

319

320

321

322

323

324

325

326

327

328

329

330

331

332

333

334

335

336

337

338

ignoring such warnings. A typical scenario that causes a warning is when the name of a pipeline parameter is found inside the task code, but the task does not have access to it, because it is not a global parameter nor defined in the set of task parameters.

One of the most notable features of the Compi workflow execution engine is that it allows a finegrained control over the execution of the pipeline tasks. While many workflow engines only allow launching the entire pipeline or partially relaunching a pipeline from a point of failure, which can also be done in Compi using the "resume" command, Compi also allows launching sub-pipelines using modifiers such as "--from", "--after", "--until" or "--before". In the example shown in Figure 4B, a combination of the "--from " and "--until" modifiers is used, resulting in the execution of all tasks in the path between "task-1" and "task-10", including "task-1" and "task-10". If the "--after" and "--before" are used instead, then "task-1" and "task10 " are not executed. A fifth modifier is "--single-task", which allows only the specified task to execute and is not compatible with the other four modifiers. The Compi documentation (https://www.sing-group.org/compi/docs/running_pipelines.html\#examples) includes several examples to illustrate how each of these options work.

Compi runs each task as a local command by default (Figure 5A). This means that if a task invokes a certain tool (e.g. a ClustalOmega alignment running "clustalo $-i /$ path/to/input.fasta $-o$ /path/to/output.fasta"), this tool must be available either from the path environment variable or by including the absolute path to the binary executable. Since dependency management is always cumbersome, a special effort has been made to offer developers and users various alternatives to deal with this problem, which are explained below.

One way to address this issue is by means of a file with a custom XML runner definition, as done in the example shown in Figure 4A with "--runners-config pipeline-runners.xml”. Individual runners are defined through a "runner" element within a runners file, where the "task" attribute is used to specify the list of tasks that the runner must execute. In this way, when a task identifier is assigned to a runner, Compi will ask the runner to run the corresponding task code (instead of running it as a local command). The usage of pipeline runners to handle dependencies allows Docker images to take responsibility for them (Figure 5D). For instance, Figure 6A shows a pipeline task named "align" that uses a tool (defined by the pipeline parameter "clustalomega") that receives a file as input and produces an output. The runner defined in Figure $6 \mathrm{~B}$ for the same task runs the specified task code (available in the environment variable "task_code") using a Docker image. The runner here is almost a generic Docker runner, and the key points are:

- First, the creation of a variable ("\$envs") with the list of parameters that must be passed as environment variables to the Docker container.

- Second, run the Docker image with the list of environment variables and mount the directory where the command has the input and output files ("workingDir" in this example).

Such a Docker runner would allow to follow an image-per-task execution pattern, where each task is executed using a different container image (Spjuth et al., 2018). An example of this execution pattern can be found in the GenomeFastScreen pipeline (https://sing- 
339

340

341

342

343

344

345

346

347

348

349

350

351

352

353

354

355

356

357

358

359

360

361

362

363

364

365

366

367

368

369

370

371

372

373

374

375

376

377

378

group.org/compihub/explore/5e2eaacce1138700316488c1), although in this case "docker run" commands are included in each task rather than provided in a runners file for the sake of simplicity. Following this image-per-task execution pattern, it is possible for a pipeline to use different versions of the same software or two tools that require different versions of some dependencies.

In addition, custom runners can also be used to submit pipeline tasks to a job scheduler such as SGE, Torque or SLURM in supercomputers or computer clusters (Figure 5B). For instance, Figure 7 shows a generic Slurm runner. Some srun parameters may need to be adjusted for each specific cluster and the "--export" parameter must be used to export all environment variables to the process to be executed, as the task parameters are declared as environment variables. In the same way, when using Compi runners, it is also possible to combine containerized execution of Compi tasks with Docker in a clustered environment, using a container orchestration system such as Kubernetes (Figure 5E).

Another way to achieve dependency management is by building a monolithic Docker image with Compi, the pipeline itself and all its dependencies (Figure 5C). This topic is explained in subsection 3.3.

Finally, dependency management can be also delegated to external systems. For example, Compi allows the use of Conda/Bioconda packages seamlessly. Each task can use them simply by activating and deactivating the corresponding Conda environments before executing the specific commands. The Metatax pipeline (https://www.singgroup.org/compihub/explore/5d807e5590flec002fc6dd83) illustrates this. For instance, Figure 8 shows the execution of a script (whose name is defined by the "validate_mapping_file" parameter, highlighted in bold) from the Qiime Bioconda package.

\section{Reproducible Application Packaging with Docker}

Compi enables the creation of portable end-user CLI applications for pipelines that can be distributed as Docker images. As noted in the previous section, this is another way to deal with dependency management, as such Docker images contain all the dependencies required by the pipeline. Pipelines distributed in this way follow an image-per-pipeline execution pattern in which all tasks are executed using the same image container (Spjuth et al., 2018) (Figure 5C), and can even be run using Docker-compatible container technologies such as Singularity. The compi-dk command-line tool is provided to assist in the development and packaging of Compi-based applications into Docker images. Pipeline development starts with the creation of a new compi-dk project with the "compi-dknew-project" command, which creates a project directory and inizialites two template files: pipeline.xml and Dockerfile. After this, the definition of the pipeline can start by modifying the pipeline.xml template and the subsequent local testing (using the compi command). Also, it can be tested by building a Docker image (using compi-dk) and running the containerized pipeline. As for the latter case, when the compi-dk build command is executed on the project directory, a Docker image for the pipeline is created. This image contains the compi executable file and a specific pipeline.xml file, along with the pipeline 
379 dependencies as defined in the Dockerfile. Figure 9 shows the Dockerfile of the MINC Computer 380 Vision pipeline for image classification based on Deep Learning (https://www.sing-

381

382

383

384

385

386

387

388

389

390

391

392

393

394

395

396

397

398

399

400

401

402

403

404

405

406

407

408

409

410

411

412

413

414

415

416

417 The Compi Hub website lists publicly available pipelines and gives access to all pipelines. When

418 a pipeline is selected, the main pipeline information is displayed, including title and description, group.org/compihub/explore/5d08a9e41713f3002fde86d5). The Dockerfile skeleton was automatically generated by compi-dk, being only necessary to add the "RUN" commands for the installation of "gluoncv" and "gnuplot" dependencies. In this regard, it is important to note that special attention was placed on the ability to derive a pipeline application image from any preexisting Docker image of interest (e.g. images with bioinformatics packages). For instance, the RNA-Seq Compi pipeline discussed above was created using the DEWE (López-Fernández et al., 2019) Docker image as a base image and the MINC Computer Vision was created using one of the Apache MXNet Docker images as a base image.

When working with a compi-dk project, it is also possible to create a Docker image for a pipeline that follows the image-per-task execution pattern. This is the case of the GenomeFastScreen pipeline (https://sing-group.org/compihub/explore/5e2eaacce1138700316488c1), in which, as explained above, a "docker run" command is included within each task instead of managing the execution of Docker using an external runner file. Regardless, most of the tasks within this pipeline run under external Docker images, thus following the image-per-task execution pattern. As the GenomeFastScreen pipeline itself is also distributed as a Docker image, it must be able to run Docker images as well (please refer to the pipeline documentation for details on how to do this).

\section{Pipeline distribution via Compi Hub}

Compi enables the creation of portable end-user CLI applications for pipelines that can be distributed as Docker images. As noted in the previous section, this is another way to deal with dependency management, as such Docker images contain all the dependencies required by the pipeline. Pipelines distributed this way follow an image-per-pipeline execution pattern in which all tasks are executed using the same image container (Spjuth et al., 2018) (Figure 5C), and can even be run using Docker-compatible container technologies such as Singularity.

Once the pipeline development is completed, it can be released through Compi Hub to increase the visibility and benefit from the Compi Hub features. Pipelines can be registered using the Compi Hub web interface (https://www.sing-group.org/compihub) or using the "compi-dk hubpush" command. Compi Hub can store several versions of a pipeline, each of them associated to a pipeline. $x m l$ file where the specific pipeline version is defined. In addition, since Compi Hub does not store either the full source code (e.g. scripts included in the pipeline.xml as source files) or the Docker images themselves, pipeline publishers are encouraged to: $(i)$ publish the source code (i.e. the compi- $d k$ project) in public repositories such as GitHub or GitLab to allow users to re-build the project locally at any time; and (ii) push the corresponding Docker image to the Docker Hub registry so that users can pull the image and follow the instructions to run the pipeline application. 
419

420

421

422

423

424

425

426

427

428

429

430

431

432

433

434

435

436

437

438

439

440

441

442

443

444

445

446

447

448

449

450

451

452

453

454

455

456

457

458

creation date, as well as links to external repositories in GitHub or Docker Hub. In addition, for each pipeline version, Compi Hub displays the following information:

- Overview: this section is headed by the pipeline DAG, generated in the backend using the "compi export-graph" command. Since it is an interactive graph, visitors can use it to navigate to each task description. Figure 10 shows the Metatax pipeline DAG (https://www.sing-group.org/compihub/explore/5d807e5590flec002fc6dd83). The DAG is followed by two tables, one containing the pipeline tasks and their associated descriptions, and a second one containing global parameters of the pipeline. Finally, this section encloses one table for each task with descriptions and specific parameters. It is important to note that all this information is automatically generated from the pipeline XML.

- Readme: this section shows the content of the README.md file when it is present in the compi-dk project. This file should be used to provide a comprehensive description of the pipeline, as well as instructions on how to use it.

- Dependencies: this section shows the content of the DEPENDENCIES.md file when it is present in the compi-dk project. We recommend that pipeline developers include this file with a human-readable description of the pipeline dependencies and the specific versions used to develop the pipeline.

- License: this section shows the content of the LICENSE file when it is present in the compi- $d k$ project. We encourage pipeline developers to include this file in their compi-dk projects so that the terms of use of the pipeline are clear.

- Dataset: this section contains a list of datasets that can be used to test the different versions of the pipeline. It is shown when a pipeline publisher associates a test dataset with the displayed pipeline. In addition to the instructions given in the Readme section, we also recommend that pipeline publishers provide test datasets to help users test the pipelines themselves.

- Runners: this section displays a list of example runner configurations when they are present in the compi- $d k$ project. Runner configurations must be stored as XML files within the "runners-example" directory of the project.

- Params: this section shows a list of example parameter configurations when they are present in the compi-dk project. Parameter configurations must be stored as plain-text key-value files in the "params-example" directory of the project.

As can be seen, Compi Hub was not designed to be a merely pipeline repository. We seek that developers accompany each pipeline with all the necessary information to ensure its portability and reproducibility by other researchers.

\section{Discussion}

Workflow management systems play a key role in the development of data science processing pipelines in multiple fields, such as bioinformatics or machine learning, among others. There are multiple solutions and approaches to develop flexible, portable, usable, maintainable and 
459 reproducible analysis pipelines in an easy way. With Compi, we progressed from a state-of-the460 art capable workflow management system to an entire application framework, focusing on the 461 transition from pipeline development to end users and the community. In this sense, we have put 462 special emphasis on providing pipelines with an advanced and automatically generated CLI, 463 which is aware of the pipeline structure, parameters and tasks descriptions, to facilitate its 464 adoption by final users. Moreover, Compi aids pipeline developers in creating all-in-one 465 distributable Docker images, as well as share the pipeline in an online automatically documented 466 hub where it will be available to the community. This combination with Docker has the added 467 benefit that Compi projects can be built into runnable CLI applications using only text files (i.e. a 468 "pipeline.xml" file, a Dockerfile, and other project files), allowing pipeline developers to use 469 control version systems to keep track of their development. Compi pipelines can be executed in 470 multiple computing layouts without any modification, from running natively on a single machine 471 to a high-performance, fully containerized cluster environment, tailored to the needs of the end 472 user. At design level, we have prioritized the use of well-known standards, such as XML, low 473 intrusiveness and language agnosticism, targeting a broader user community. In this sense, we 474 avoid defining pipelines via DSLs based on a specific programming language, forcing a scripting 475 language to define each task code, or be coupled to specific dependency management systems, 476 such as Python (Conda), R (Cran) or Java (Maven).

477 Unlike Snakemake, the Nextflow and SciPipe tools allow dynamic scheduling, that is, the ability 478 to change the pipeline structure dynamically to schedule a different number of tasks based on the 479 results of a previous step or any other parameter. Compi allows dynamic scheduling via $(i)$ the 480 "if" attribute of tasks, which executes a command just before the task is about to run, allowing 481 the task to be skipped dinamically, and ( $i$ i) foreach loops, which can take their iteration values 482 from the output of a command, which is executed just before the foreach loop is about to run, 483 allowing, for instance, to do more parallel iterations depending on the number of files generated 484 by a previous task.

485 Compi, Snakemake, and Nextflow are language independent, allowing external scripts written in 486 any programming language to be invoked.

487 Similar to Compi runners, Nextflow defines executors, which are the components that determine 488 where a pipeline process runs and its execution is supervised. It provides multiple built-in 489 executors to manage execution on SGE, SLURM, Kubernetes, and many others. In SciPipe, this 490 can be achieved by using the Preprend field when defining processes, similarly to how Compi 491 runners work.

492 Regarding containerization, as explained above, when pipeline tasks need to run in isolated 493 containers, Compi users must include the corresponding command (e.g. "docker run") in the task 494 code or in a runner that tackles the execution of such tasks. Additionally, pipeline developers can 495 create a Docker image for the entire pipeline so that all tasks are executed in the same container. 496 In both cases, the developer must mount the paths to the input and output files of each task when 497 using Docker. In this sense, Nextflow provides built-in support to run individual tasks or 498 complete pipelines using Docker, Singularity or Podman images. In the case of Docker, 
499 Nextflow is able to mount the input and output paths automatically, since it is aware of the files

500 needed by tasks. Similarly, Snakemake has built-in support to execute complete pipelines on

501 Docker images and individual rules in isolated Conda environments. The SciPipe documentation 502 does not provide information on how to containerize pipelines.

503 Logging is another important feature of workflow management systems, allowing pipeline users 504 to see how execution went and determine causes of errors if necessary. SciPipe has been 505 designed with special care on logging and collecting metadata about each executed task. 506 Following a data-centric audit logging approach, SciPipe generates a JSON file for each output 507 file that contains the full trace of the tasks that were executed to generate it. Nextflow provides a 508 log command that returns useful information about a specific pipeline execution, and

509 incorporates a "--with-report" option in the run command that instructs Nextflow to generate an 510 HTML execution report that includes many useful metrics about a specific workflow execution. 511 It also supports a "--with-trace" option in the run command that generates an execution trace file 512 that contains useful information about each process executed as part of the pipeline (e.g. 513 submission time, start time, completion time, CPU, and memory usage). As previously stated, 514 the "compi run" command provides the "--logs" option to specify a directory to save the stdout 515 and stderr outputs and the specific parameter values of each task execution in separated files 516 prefixed with the corresponding task name.

517 Nextflow has nf-core, an environment with a dual purpose: to provide an online repository of 518 Nextflow pipelines and to provide a command-line tool to interact with the repository and 519 manage the execution of the hosted pipelines. Similarly, the Compi Hub repository allows users 520 to discover and explore pipelines, and the compi- $d k$ tool allows to push pipelines to the hub via 521 command line. Snakemake does not provide a similar public repository, but a dedicated GitHub 522 project exists (https://github.com/snakemake-workflows/docs).

523 After these considerations, and given the choices available, we believe that Compi may be a 524 reasonable choice for researchers with CLI skills looking to create medium complexity pipelines 525 without requiring them to learn new programming languages (e.g. to learn the Nextflow DSL or 526 Go for SciPipe pipeline development). In this way, researchers would benefit from the common 527 features of the workflow management engine that Compi offers without the need for much 528 training. In addition, thanks to the auto-generated CLI, Compi would be the most suitable 529 solution for those pipelines meant to be used by researchers as end-user applications. As the 530 existing pipeline examples presented in the previous section demonstrate, pipeline-based 531 applications developed in this way can easily be distributed as Docker images. Finally, to 532 illustrate the way of creating Compi pipelines and the main differences with other workflow 533 management systems, we have implemented a Nextflow example pipeline in Compi

534 (Supplementary File 2). This simple example, the description made in the previous section, and 535 the public pipelines available on Compi Hub, will allow potential users to determine when 536 Compi may be the most suitable option.

537

\section{Conclusions}


539 Compi is an application framework for developing pipeline-based end-user applications in

540 bioinformatics and data science. Two Compi design principles are low intrusiveness and

541 language agnosis, with the goal of covering a wide variety of scenarios and providing the most

542 flexibility to pipeline developers. Noteworthy, Compi pipelines can be executed in multiple

543 computing layouts without the need to modify the pipeline definition, from running natively on a

544 single machine to a fully-containerized, high-performance cluster environment, tuned for the

545 needs of the end user.

546 To complement the Compi workflow execution engine, we have also created Compi

547 Development Kit (compi-dk) and Compi Hub. Thanks to the Compi Development Kit, pipelines

548 can be packaged as self-contained Docker images that also include pipeline dependencies, and

549 can be shared publicly with minimal effort using Compi Hub.

550 Future work include, among other tasks, the following issues: $(i)$ improving the metadata section

551 of the pipelines to allow the inclusion of more information (e.g. licensing, attributions, or custom

552 information); (ii) including the possibility of creating stackable runners that gives more

553 flexibility and power to customize task execution; and (iii) enhancing the logging reports

554 generated by Compi.

555

556 Acknowledgements

557 SING group thanks the CITI (Centro de Investigación, Transferencia e Innovación) from the

558 University of Vigo for hosting its IT infrastructure.

559

560

\section{References}

561 Afgan E, Baker D, Batut B, van den Beek M, Bouvier D, Čech M, Chilton J, Clements D, Coraor

562

N, Grüning BA, Guerler A, Hillman-Jackson J, Hiltemann S, Jalili V, Rasche H, Soranzo

563

N, Goecks J, Taylor J, Nekrutenko A, Blankenberg D. 2018. The Galaxy platform for

564 accessible, reproducible and collaborative biomedical analyses: 2018 update. Nucleic

565 Acids Research 46:W537-W544. DOI: 10.1093/nar/gky379.

566 Di Tommaso P, Chatzou M, Floden EW, Barja PP, Palumbo E, Notredame C. 2017. Nextflow

567 enables reproducible computational workflows. Nature Biotechnology 35:316-319. DOI:

568 10.1038/nbt.3820.

569

Graña-Castro O, López-Fernández H, Fdez-Riverola F, Al-Shahrour F, Glez-Peña D. 2020a.

570 Proposal of a New Bioinformatics Pipeline for Metataxonomics in Precision Medicine. In: Fdez-Riverola F, Rocha M, Mohamad MS, Zaki N, Castellanos-Garzón JA eds. 
572

573

574

575

576

577

578

579

580

581

582

583

584

585

586

587

588

589

590

591

592

593

594

Practical Applications of Computational Biology and Bioinformatics, 13th International

Conference. Cham: Springer International Publishing, 8-15. DOI: 10.1007/978-3-03023873-5_2.

Graña-Castro O, López-Fernández H, Nogueira-Rodríguez A, Fdez-Riverola F, Al-Shahrour F, Glez-Peña D. 2020b. Metatax: Metataxonomics with a Compi-Based Pipeline for Precision Medicine. Interdisciplinary Sciences: Computational Life Sciences 12:252_257. DOI: $10.1007 / \mathrm{s} 12539-020-00368-6$.

Grüning B, Chilton J, Köster J, Dale R, Soranzo N, van den Beek M, Goecks J, Backofen R, Nekrutenko A, Taylor J. 2018. Practical Computational Reproducibility in the Life Sciences. Cell Systems 6:631-635. DOI: 10.1016/j.cels.2018.03.014.

Köster J, Rahmann S. 2012. Snakemake—a scalable bioinformatics workflow engine. Bioinformatics 28:2520-2522. DOI: 10.1093/bioinformatics/bts480.

Lampa S, Dahlö M, Alvarsson J, Spjuth O. 2019. SciPipe: A workflow library for agile development of complex and dynamic bioinformatics pipelines. GigaScience 8. DOI: 10.1093/gigascience/giz044.

Leipzig J. 2017. A review of bioinformatic pipeline frameworks. Briefings in bioinformatics 18:530-536. DOI: 10.1093/bib/bbw020.

López-Fernández H, Blanco-Míguez A, Fdez-Riverola F, Sánchez B, Lourenço A. 2019. DEWE: A novel tool for executing differential expression RNA-Seq workflows in biomedical research. Computers in Biology and Medicine 107:197-205. DOI:

10.1016/j.compbiomed.2019.02.021.

López-Fernández H, Duque P, Vázquez N, Fdez-Riverola F, Reboiro-Jato M, Vieira CP, Vieira J. 2020. Inferring Positive Selection in Large Viral Datasets. In: Fdez-Riverola F, Rocha 
595

596

597

598

599

600

601

602

603

604

605

606

607

608

609

610

611

612

613 Sadedin SP, Pope B, Oshlack A. 2012. Bpipe: a tool for running and managing bioinformatics

614

615

616

M, Mohamad MS, Zaki N, Castellanos-Garzón JA eds. Practical Applications of Computational Biology and Bioinformatics, 13th International Conference. Cham:

Springer International Publishing, 61-69. DOI: 10.1007/978-3-030-23873-5_8.

López-Fernández H, Vieira CP, Fdez-Riverola F, Reboiro-Jato M, Vieira J. 2021. Inferences on Mycobacterium Leprae Host Immune Response Escape and Antibiotic Resistance Using Genomic Data and GenomeFastScreen. In: Panuccio G, Rocha M, Fdez-Riverola F, Mohamad MS, Casado-Vara R eds. Practical Applications of Computational Biology \& Bioinformatics, 14th International Conference (PACBB 2020). Advances in Intelligent Systems and Computing. Cham: Springer International Publishing, 42-50. DOI: 10.1007/978-3-030-54568-0_5.

Nogueira-Rodríguez A, López-Fernández H, Graña-Castro O, Reboiro-Jato M, Glez-Peña D. 2021. Compi Hub: A Public Repository for Sharing and Discovering Compi Pipelines. In: Panuccio G, Rocha M, Fdez-Riverola F, Mohamad MS, Casado-Vara R eds. Practical Applications of Computational Biology \& Bioinformatics, 14th International Conference (PACBB 2020). Advances in Intelligent Systems and Computing. Cham: Springer International Publishing, 51-59. DOI: 10.1007/978-3-030-54568-0_6.

Perkel JM. 2019. Workflow systems turn raw data into scientific knowledge. Nature 573:149150. DOI: $10.1038 / \mathrm{d} 41586-019-02619-\mathrm{z}$. pipelines. Bioinformatics 28:1525-1526. DOI: 10.1093/bioinformatics/bts167.

Spjuth O, Capuccini M, Carone M, Larsson A, Schaal W, Novella J, Di Tommaso P, Notredame C, Moreno P, Khoonsari PE, Herman S, Kultima K, Lampa S. 2018. Approaches for 


\section{Figure 1}

Lifecycle of developing, packaging, distributing, and running Compi-based applications.

For developers, this lifecycle comprises three main stages: pipeline development (including testing the pipeline as pipeline user), application packaging, and distribution. 


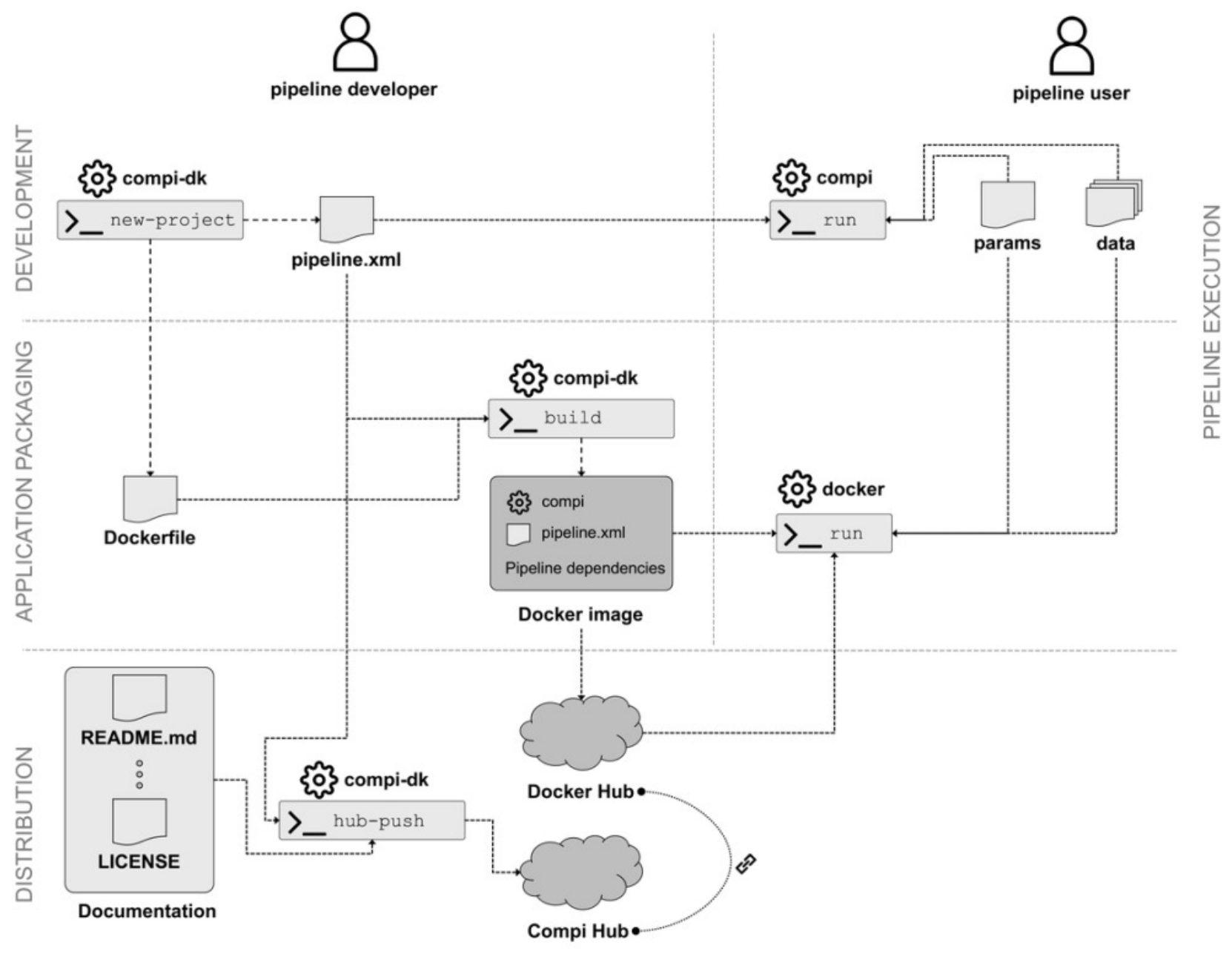




\section{Figure 2}

Minimal pipeline example of a Compi pipeline with two tasks.

A specific section ("params") defines two parameters ("name" and "output"), used in the two pipeline tasks, named "greetings" and "bye". Both tasks print the value of the "name" parameter to the path specified in the "output" parameter. By default, tasks are executed as Bash commands and this is the case of the "greetings" task. The "bye" task is written in Perl and the "interpreter" parameter of the task definition indicates how to invoke the Perl interpreter to run the task code.

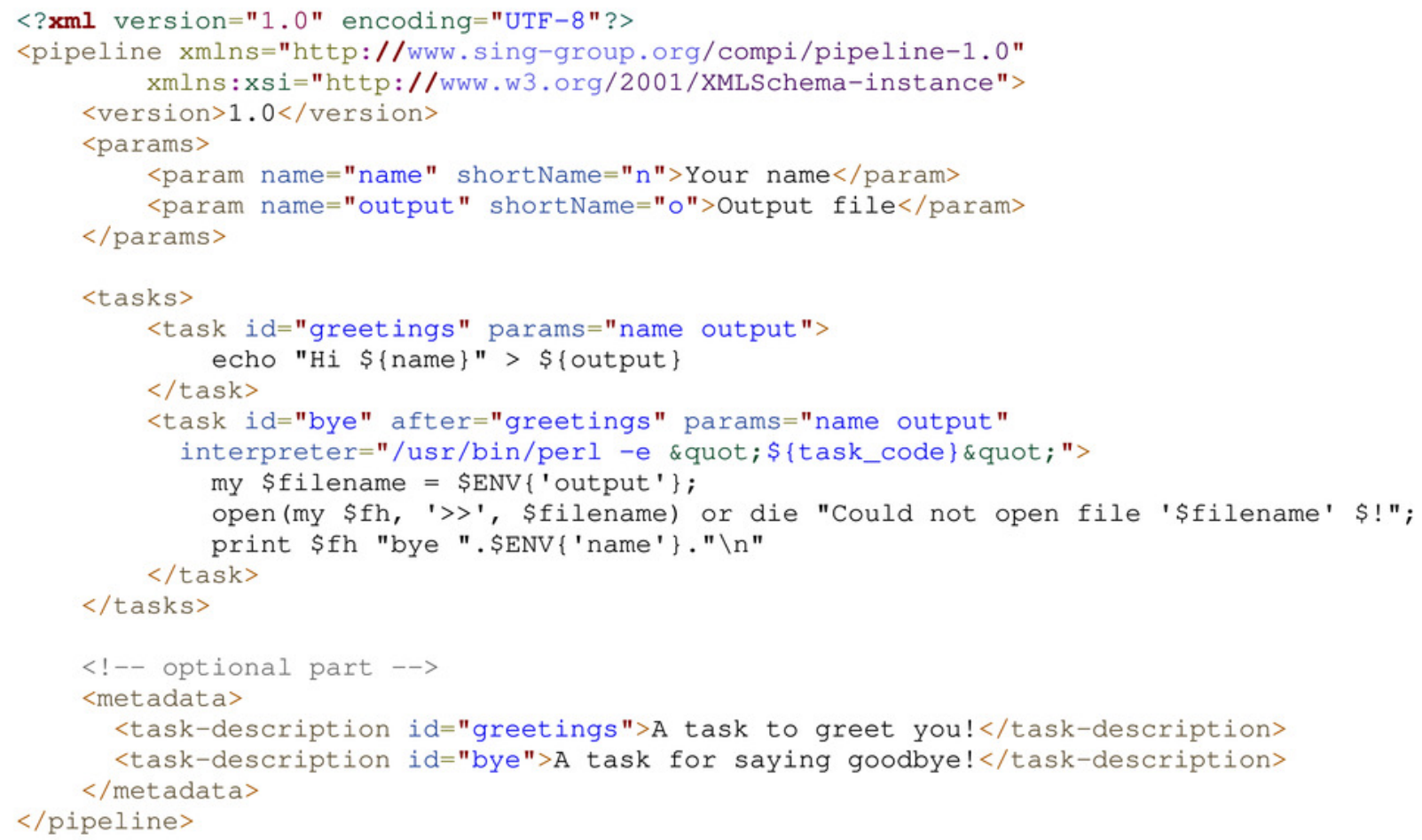




\section{Figure 3}

Example of Compi pipeline using iterative foreach tasks.

Note the "*" character when the dependency of the "analyze" task on the "preprocess" task is defined. This way, the second foreach is binded to the first one, meaning that the two foreach tasks iterate over the same collection of elements and allowing Compi to start the execution of each iteration of the second foreach right after the corresponding iterations of the first foreach had finished.

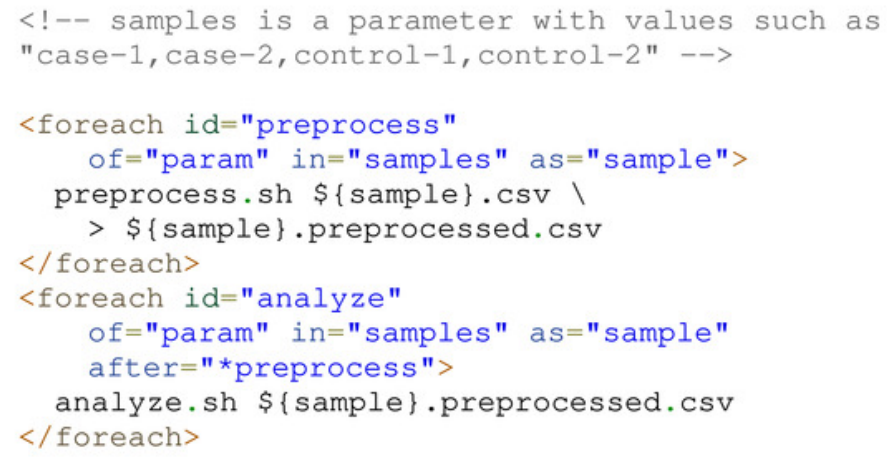




\section{Figure 4}

Examples of Compi parameters to control how pipelines must be executed.

Compi parameters belong to three main categories: pipeline inputs (i.e. the pipeline definition and its input parameters), logging, and execution control (i.e. specify whith tasks must be executed). 
A) compi run

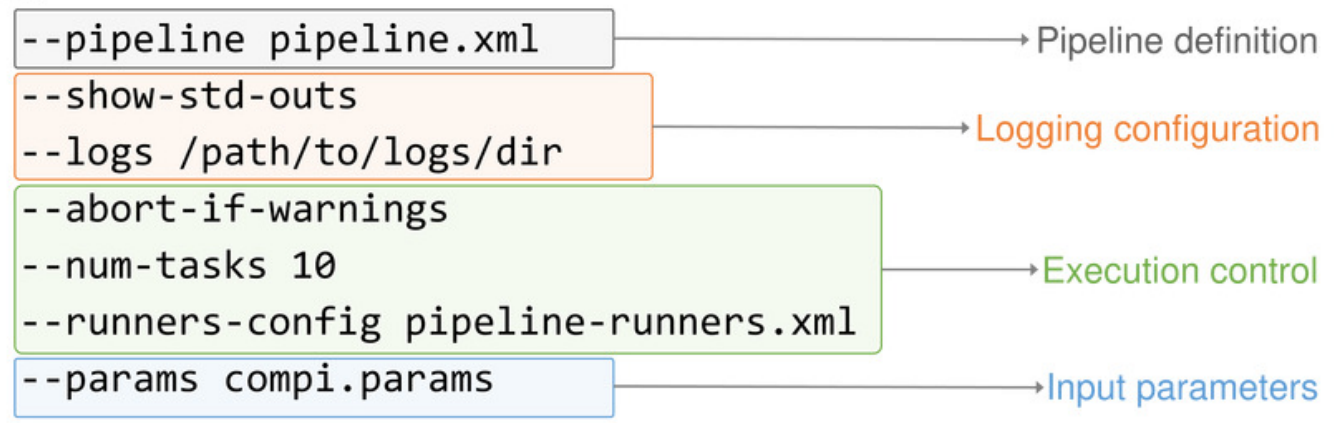

B) compi run

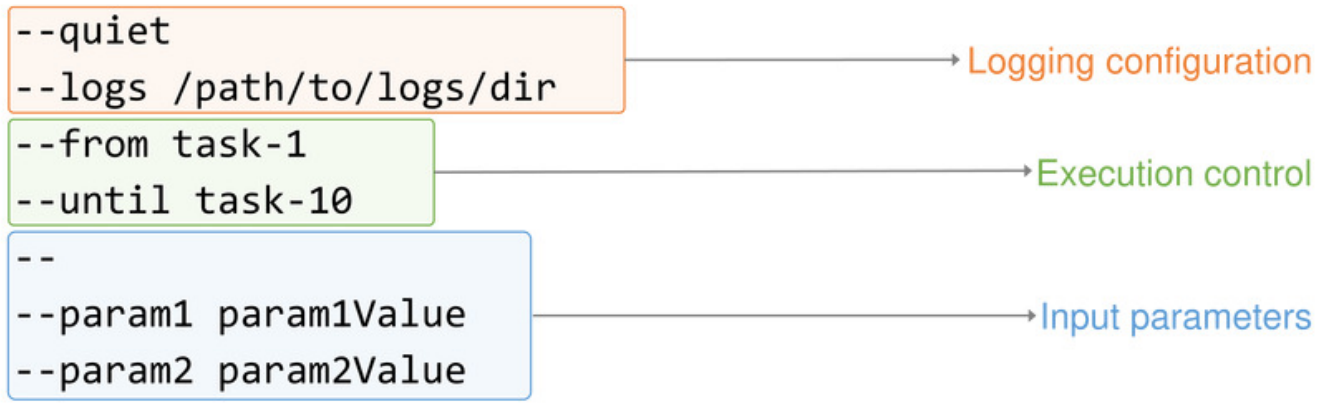




\section{Figure 5}

Different ways of executing tasks involving external dependencies in Compi.

The upper left quadrant shows the simplest case (scenario A), where the pipeline is run on a single machine and external dependencies must be available on the system. One way to manage external dependencies is by using containers like the one shown in the bottom left quadrant. While scenario $\mathrm{C}$ shows the case of a Compi application which runs in a container with all dependencies, scenario D shows a Compi application that uses a Docker image for running the external dependencies. Custom Compi runners can also be used to submit tasks into an HPC cluster, as scenario B shows. Finally, it is also possible to combine containerized execution of Compi tasks with Docker in a clustered environment by using a container orchestration system such as Kubernetes. 

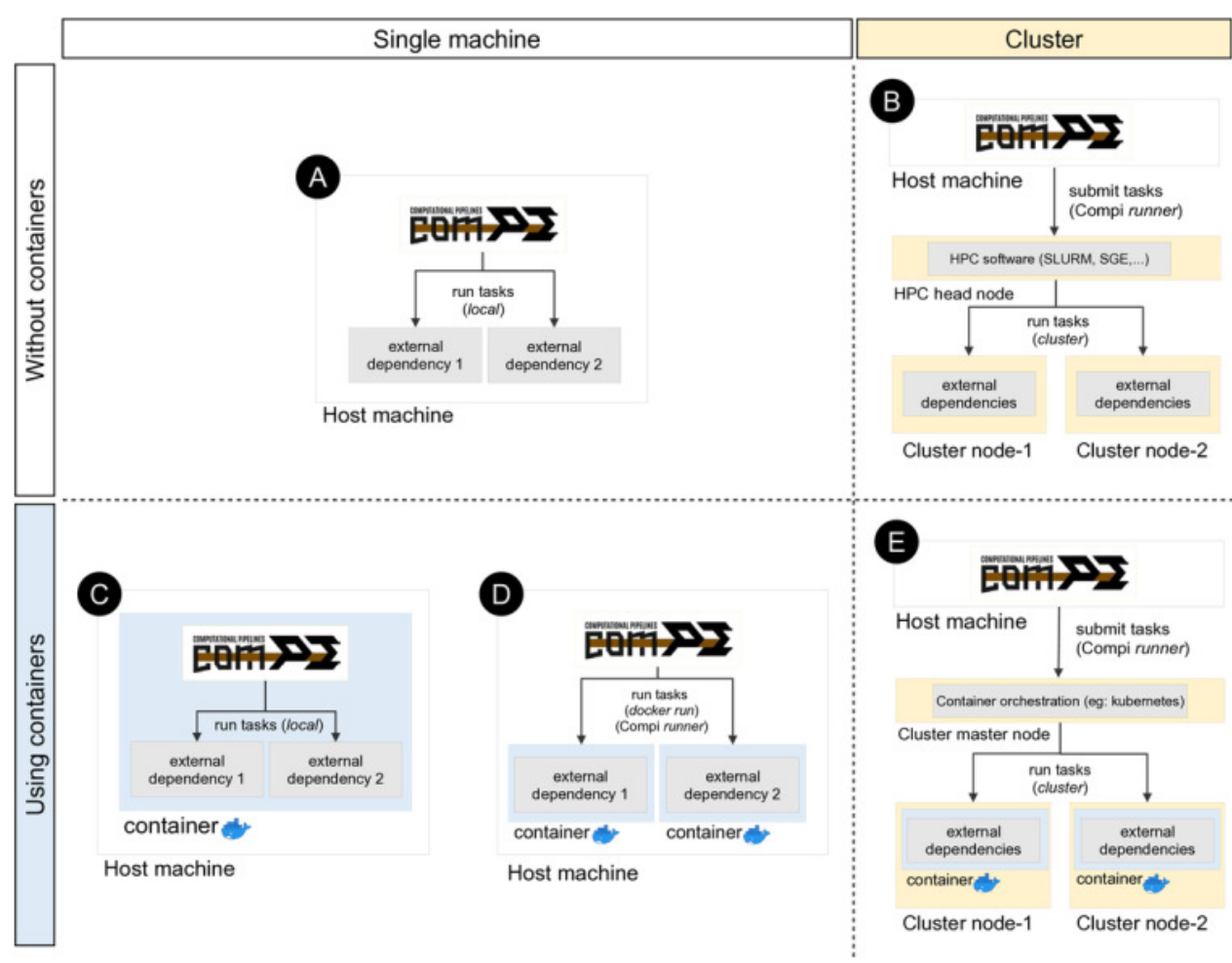


\section{Figure 6}

Use of custom Compi runners.

(A) Task of a Compi pipeline that invokes a command defined in the "clustalomega" pipeline parameter. This command must be available in the system. (B) Example of Compi runner for the "align" task that runs the task source code (available in the "task_code" environment variable) using a Docker image that has the command installed.

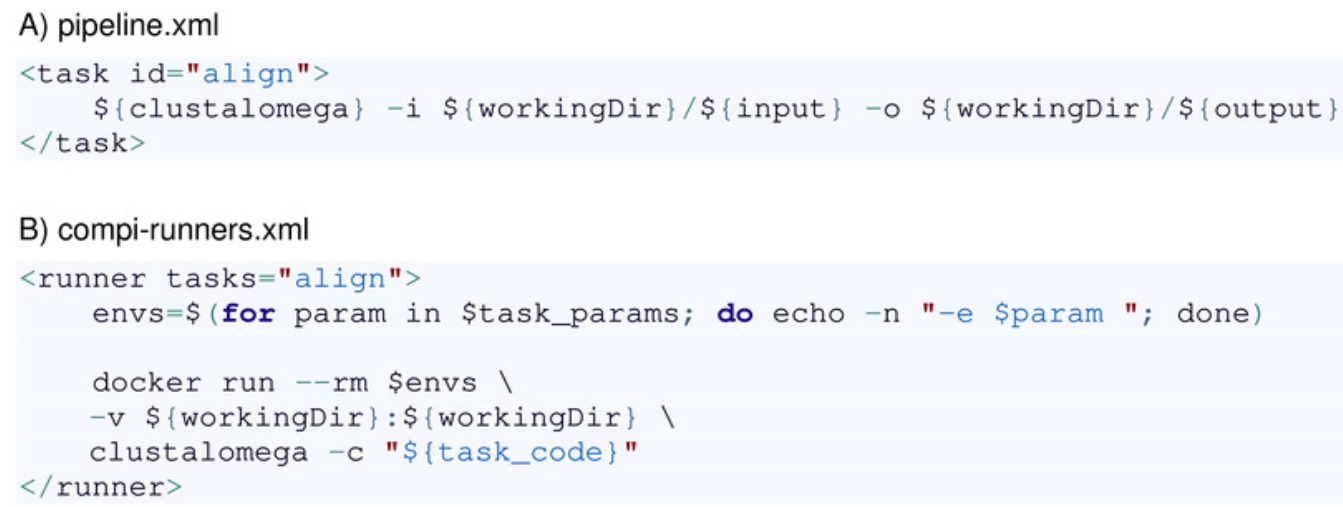




\section{Figure 7}

\section{Generic Compi runner for submitting tasks into a Slurm system.}

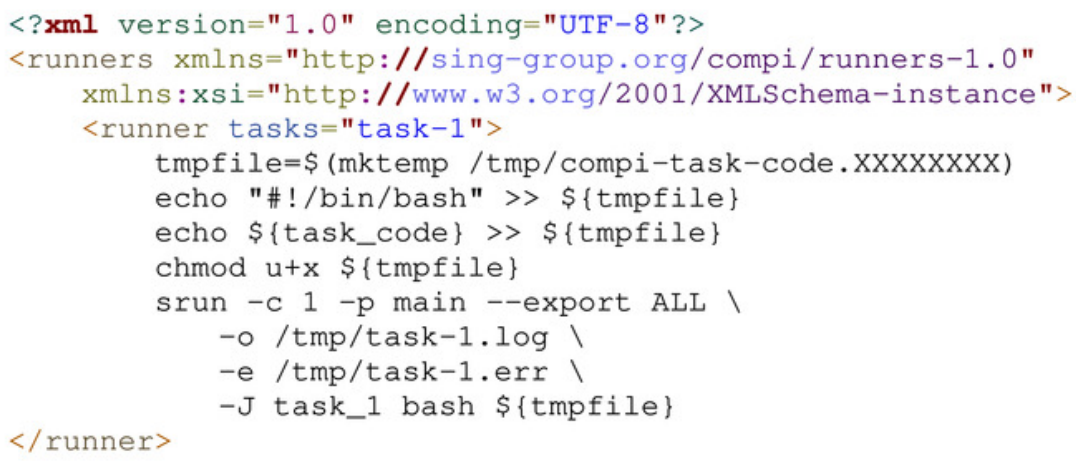




\section{Figure 8}

Dependency management in Compi pipelines using Conda/Bioconda packages.

In this example from the Metatax pipeline, the "validate_mapping" task activates the qiime1 environment to load the Qiime Bioconda package, executes a script from this environment, and finally deactivates the environment. Each task can use them by simply activating and deactivating the corresponding environments before executing the specific commands.

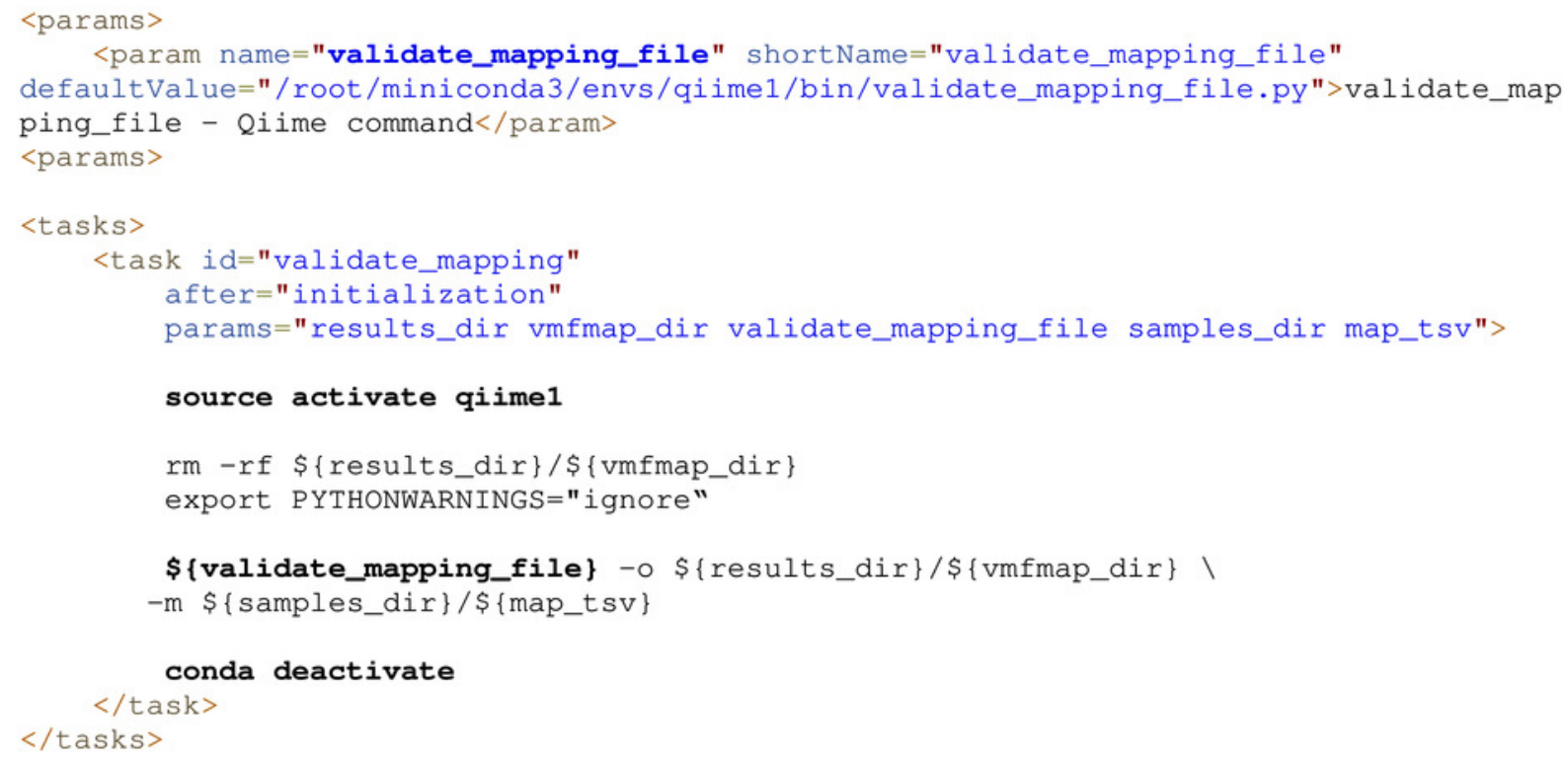




\section{Figure 9}

\section{Docker image for the MINC Computer Vision pipeline (https://sing-group.org/compihub/explore/5d08a9e41713f3002fde86d5).}

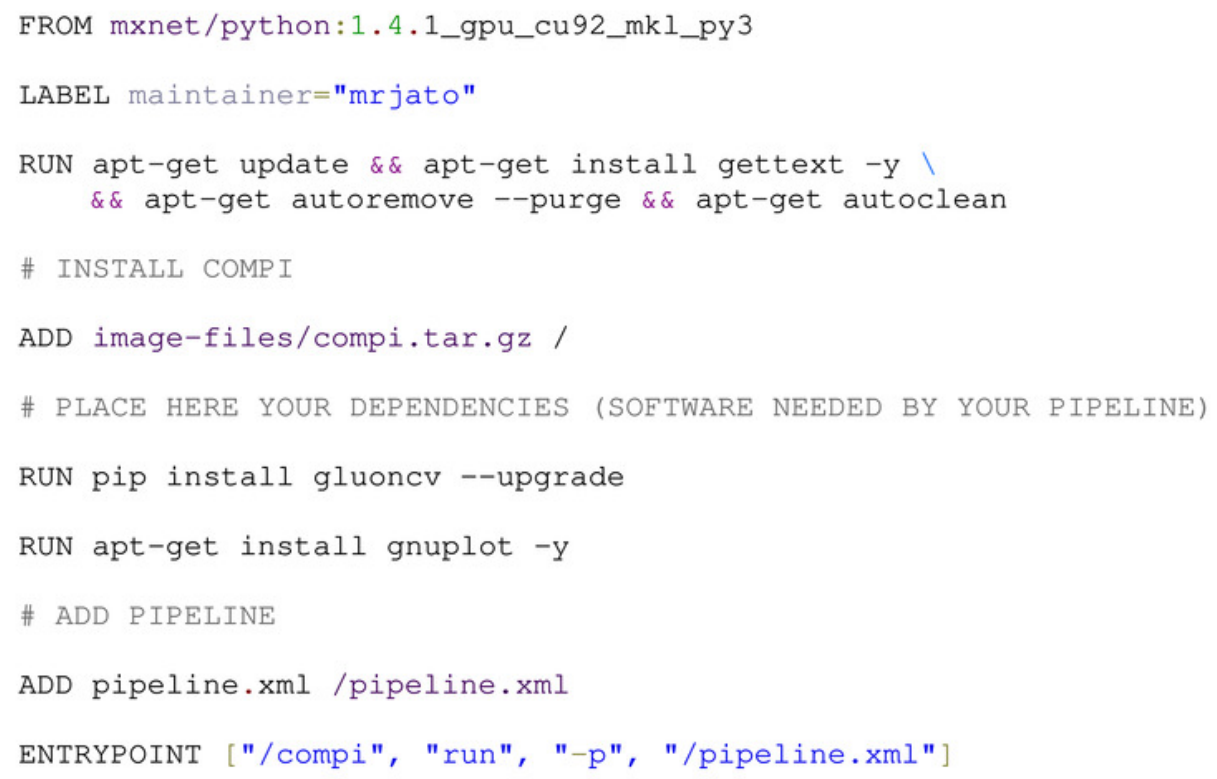




\section{Figure 10}

DAG of Metatax as shown in Compi Hub (https://www.sing-group.org/compihub/explore/5d807e5590flec002fc6dd83).

Compi Hub automatically generates it by running the "compi export-graph" command in the backend. Foreach tasks are drawn with a dashed border to differentiate them from regular tasks.

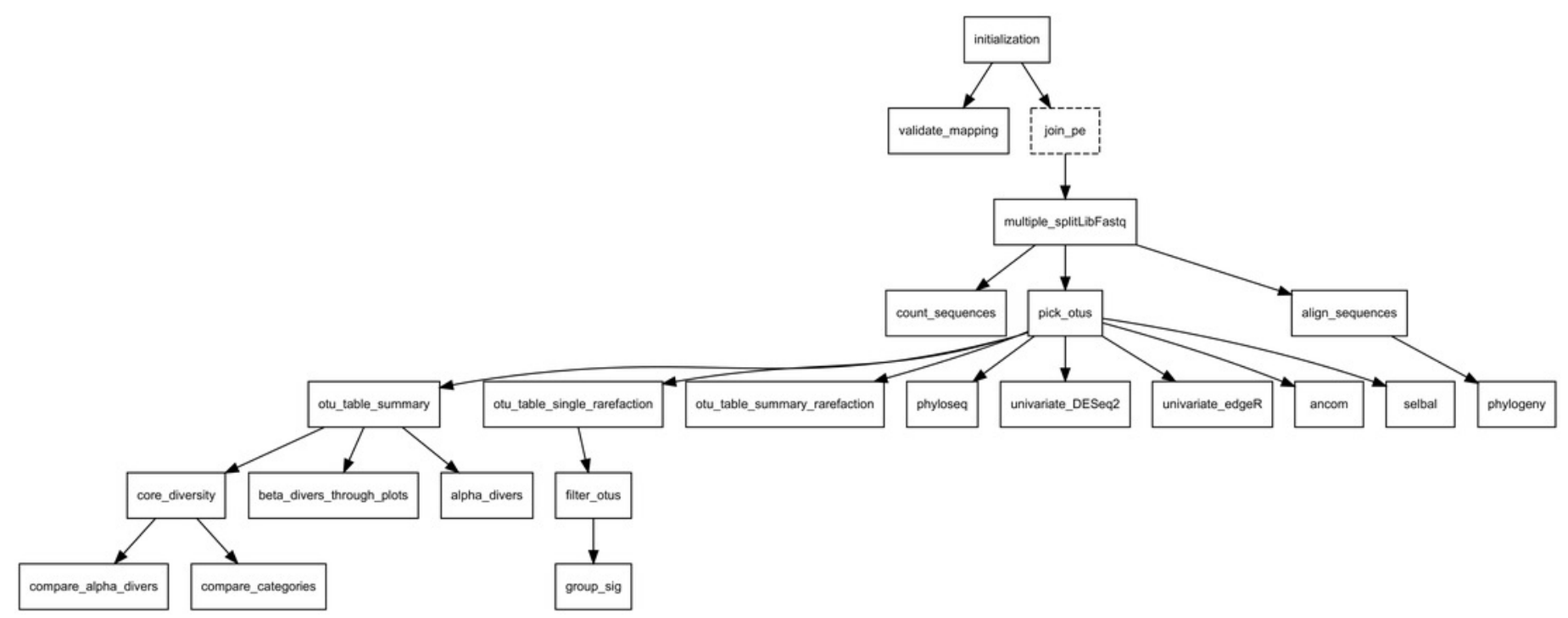

